\title{
ON THE EXTREME POINTS OF THE INTERVAL BETWEEN TWO OPERATORS ${ }^{1}$
}

\author{
FRANCIS J. NARCOWICH
}

\begin{abstract}
Given that $A, B$ are operators on a complex Hilbert space, and that $B-A$ is nonnegative, the interval between $A$ and $B$ consists of every operator, $G$, such that both $B-G$ and $G-A$ are nonnegative. The extreme points of such an interval are exhibited and the interval is shown to be the closure of the convex hull of these extreme points in the weak-operator topology.
\end{abstract}

Let $\mathcal{H}$ be a complex Hilbert space and let $B(\mathcal{H})$ be the set of bounded linear operators on $\mathcal{H}$. If $A$ and $B$ belong to $B(\mathcal{H})$ and $C^{2}=B-A \geqslant 0$, $C \geqslant 0$, in the usual operator sense, the interval between $A$ and $B$ is the set

$$
I(A, B)=\{G \in B(\mathcal{H}) \mid B-G \geqslant 0 \text { and } G-A \geqslant 0\} .
$$

This set is obviously convex. What are its extreme points? Is $I(A, B)$ the closure of the convex hull of its extreme points in one of the operator topologies?

The two questions above arise in the study of the range of the Stieltjes transform over all operator-valued measures which generate a given Stieltjes Hermitian moment sequence (cf. [3]).

The standard operator-theoretic notation used in the paper is the same as in [1].

The main result is the

THEOREM. Let $P$ be the orthogonal projection onto $\operatorname{Range}(C)^{-} . E$ is an extreme point of $I(A, B)$ if and only if

$$
E=A+C Q C,
$$

where $Q$ is a projection in $I(0, P)$. Moreover, $I(A, B)$ is the closure of the convex hull of its extreme points in the weak operator topology.

The proof will be given later. Note that $A$ and $B$ are not assumed positive, or even selfadjoint, just the difference $B-A$ is assumed to be a nonnegative operator, $C^{2}$.

The proof of the Theorem requires the identity

$$
I(A, B)=A+I\left(0, C^{2}\right)
$$

Received by the editors January 31, 1977 and, in revised form, May 3, 1977.

AMS (MOS) subject classifications (1970). Primary 47D20; Secondary 52A40, 52A05.

Key words and phrases. Interval between two operators, extreme points, convex hull.

1 This work is partly supported by NSF Grant No. MCS76-06631. 
which follows directly from the definition of $I(A, B)$, and the

LEMMA. With $P$ as in the Thoerem, $G \in I\left(0, C^{2}\right)$ if and only if there exists $M \in I(0, P)$ such that $G=C M C$. Moreover, $M$ is uniquely determined by $G$.

Proof. If $M \in I(0, P)$ and $G=C M C$, a straightforward calculation shows that $G \in I\left(0, C^{2}\right)$. Conversely, if $G \in I\left(0, C^{2}\right)$, a theorem of Douglas (cf. [1]) implies the existence of a unique $Z \in B(\mathcal{H})$ with the following. properties: (a) $G^{1 / 2}=C Z$; (b) $P Z=Z$; (c) $\|Z\| \leqslant 1$; and (d) $\operatorname{Null}(Z)=$ $\operatorname{Null}\left(G^{1 / 2}\right)$. With $M=Z Z^{*}$, from (a) it follows that $G=C M C$, and from (b) and (c) that $M \in I(0, P)$.

Suppose that $M^{\prime} \in I(0, P)$ and $C M^{\prime} C=C M C$. By forming an inner product, using $C=C^{*}$, and then taking limits, it follows that for every $\phi$, $\psi \in \operatorname{Range}(C)^{-},(\psi, M \phi)=\left(\psi, M^{\prime} \phi\right)$. This is equivalent to

$$
P M P=P M^{\prime} P \text {. }
$$

On the other hand, if $N \in I(0, P)$, then $\operatorname{Null}(N) \subseteq N u l l(P)$ and, hence, $N(I-P)=0$. This and the adjoint equation give $N P=P N=N$. The last equation and (4) imply that $M=M^{\prime}$.

Proof of the Theorem. For every $G \in I\left(0, C^{2}\right)$, let $M(G)$ be the unique element of $I(0, P)$ such that $G=C M(G) C$. The bijective map $G \rightarrow M(G)$ preserves convex combinations: If $G=\alpha G_{1}+\beta G_{2}, \alpha+\beta=1, \alpha \geqslant 0, \beta \geqslant$ 0 , then $G=C\left(\alpha M\left(G_{1}\right)+\beta M\left(G_{2}\right)\right) C$. Since $I(0, P)$ is convex, $\alpha M\left(G_{1}\right)+$ $\beta M\left(G_{2}\right) \in I(0, P)$. By the Lemma, $M(G)=\alpha M\left(G_{1}\right)+\beta M\left(G_{2}\right)$. The implication is that $G$ is an extreme point of $I\left(0, C^{2}\right)$ if and only if $M(G)$ is an extreme point of $I(0, P)$.

What are the extreme points of $I(0, P)$ ? If $B(P \mathcal{H})$ is regarded as a $C^{*}$-algebra, with $P$ the identity, then $I(0, P)$ is the intersection of the positive elements in $B(P \mathcal{H})$ with the closed unit ball. The extreme points of such an intersection are just the projections (cf. Sakai [4, Proposition 1.6.2]) and, hence, the extreme points of $I\left(0, C^{2}\right)$ are all of the form $C Q C$, where $Q$ is a projection in $I(0, P)$. This fact and (3) imply the extreme points of $I(A, B)$ are of the form (2).

The set $I(A, B)$ is compact in the weak operator topology because it is obviously closed and can be placed in a compact set, a closed ball of sufficiently large radius [2, p. 512]. By the Krein-Milman theorem (cf. [2]), $I(A, B)$ is the closure of the convex hull of its extreme points in this topology.

The reason for using a complex Hilbert space is that, in the real case, the positivity of an operator no longer guarantees its selfadjointness. By modifying the definition of the interval between two operators so as to include only selfadjoint operators, it is possible to extend the results given to the real case. The modifications necessary to do this are minor, mostly technical, and have been omitted.

Acknowledgement. The author wishes to thank Professor J. Ward for helpful suggestions. 


\section{REFERENCES}

1. R. G. Douglas, On majorization, factorization and range inclusion of operators on Hilbert space, Proc. Amer. Math. Soc. 17 (1966), 413-415.

2. N. Dunford and J. T. Schwartz, Linear operators. Part I, Interscience, New York, 1967.

3. F. J. Narcowich, $R$-operators. II: On the approximation of certain operator-valued analytic functions and the Hermitian moment problem, Indiana Univ. Math. J. 26 (1977), 483-513.

4. S. Sakai, $C^{*}$-algebras and $W^{*}$-algebras, Springer-Verlag, Berlin and New York, 1971.

Department of Mathematics, Texas A\&M University, College Station, TeXas 77843 